\title{
Original CLINICAL USE OF ELECTRONIC PORTAL IMAGING (EPID) NEMROCK Article EXPERIENCE SINGLE INSTITUTION EXPERIENCE
}

\author{
Mohamed Abdulla ${ }^{1}$ and Mohamed Galal ${ }^{2}$ \\ ${ }^{1}$ Clinical Oncology, Kasr El-Aini School of Medicine,Cairo University ${ }^{2}$ Radiation Medical Physics \\ Unit, Clinical Oncology, Kasr El-Aini School of Medicine, Cairo University.
}

\section{ABSTRACT}

Introduction: Radiation therapy can only be effective if the intended dose is delivered as prescribed throughout the treatment course. Most of modern radiation therapy machines are equipped with Electronic Portal Imaging Device (EPID) with sufficient contrast resolution to image bony anatomy. Setup verification and errors detection are proved to be major determinants of treatment accuracy.

Patients and Methods: Ten patients with brain tumors scheduled for conformal radiation treatment were evaluated for setup errors via the use of amorphous silicon electronic portal imaging device

Results: The variations of isocenter shift throughout treatment course ranged from 0.732.50- mm and 0.403.00- mm (Anterior Longitudinal and Lateral Shifts), and 0.404.00- $\mathrm{mm}$ for lateral fields.

Conclusion: EPID is a powerful tool to improve overall treatment accuracy in patients with brain tumors treated with conformal radiation therapy.

Key Words: Conformal radiation therapy, EPID, brain tumors.

Corresponding Author: Mohamed Abdulla: E-mail.: Mohamedabdulla2001@yahoo.com

\section{INTRODUCTION}

Radiation therapy can only be effective if the intended dose is delivered as prescribed throughout the treatment course. Electronic Portal Imaging (EPID) is here to stay and is now available as a standard equipment with virtually all state of the art treatment machines from every major manufacturer and some third parties. These devices have sub-millimeter spatial and sufficient contrast resolutions (superior to films) to image most bony anatomy or a small metallic marker implanted in tissues. The American Association of Physics in Medicine formed a Task Group (TG58) on clinical implementation of EPID Technology which outlined the fundamental operating characteristics and its incorporation into routine daily practice ${ }^{1,2}$.

Setup verification and error detection tools allow the EPID to become an effective aid to verification of patient setup and target localization with respect to the treatment geometry especially when complicated techniques are used e.g. 3DCRT or IMRT, to be sure that the patient's tumor is receiving the accurate dose to guard against under or overdose, the organs at risk to receive the accep Table dose and their patient fixation system is trus Table. Proper evaluation of treatment setup involves relating the information in a portal image to that extracted from a reference data from simulation and planning ${ }^{3}$.

Figure (1) graphically depicts how both systematic and random target localization errors are taken into account when determining margins. Each star in the fig. represents a target localization position (isocenter shift) for a single fraction measured in the superior-inferiorand lateral directions. Each group of stars represents the data for an individual patient. The systematic localization error for each patient is represented by the average of the stars indicated by straight dashed arrows. The random error for each patient is represented by the distribution of the stars about the average shown by the dashed line ellipses (Fig. 1-A). (Figures (1-B and 1-C) show if both systematic and random errors are eliminated or reduced, the margin can be made individually small ${ }^{4,5}$.

Correction methods to identify and reduce systematic and random localization errors can be divided into offline and online protocols. Offline correction entails acquiring, reviewing and analyzing image data, to be acted upon at a later time. Complex offline correction models have been evolved to allow for treatment verification for complex treatments without a large increase in time or cost for the information based on establishing error thresholds derived from a patient population for specific treatment sites. EPID is one of the most common offline setup verification tools ${ }^{6,7}$.

Online correction strategies are aimed at acquiring image data, doing an analysis and taking an action during the fraction the patient is being treated. Studies indicated that up to $50 \%$ of initial fields are judged in error and corrected. Moreover, final offline review suggested that individual setup error remained ${ }^{8,9}$. 


\section{PATIENTS AND METHODS}

The current work is a prospective study carried out in Department of Clinical Oncology and Nuclear Medicine, Kasr El-Aini School of Medicine, Cairo University during March and April 2008, where 10 patients with diversity of brain tumors (Gliomas and Meningiomas) scheduled for postoperative or radical conformal irradiation therapy were assessed for accuracy of treatment setup and radiation volume variations along their treatment course via the application of electronic portal imaging technology (EPID) to ensure adherence to pre-determined treatment specifications.
Electa Precise Linear Accelerator was used for treatment of study. It is supplied with multi-leaf collimator (MLC) consisting of 40 leaf pairs, having a maximum field size of $40 \mathrm{X} 40 \mathrm{~cm}^{2}$. Amorphous Silicon electronic portal image device (iView GT 3.2, Electa Oncology Systems, Crawley, UK) was used for treatment monitoring and setup verification. EPID is consisting of a front metal sheet to convert X-rays to light. The light is detected using an array of $\mathrm{Si}: \mathrm{H}$ photodiodes controlled by Si:H TFT . The photodiodes are electronically read and form the pixels of the image (Fig. 2).

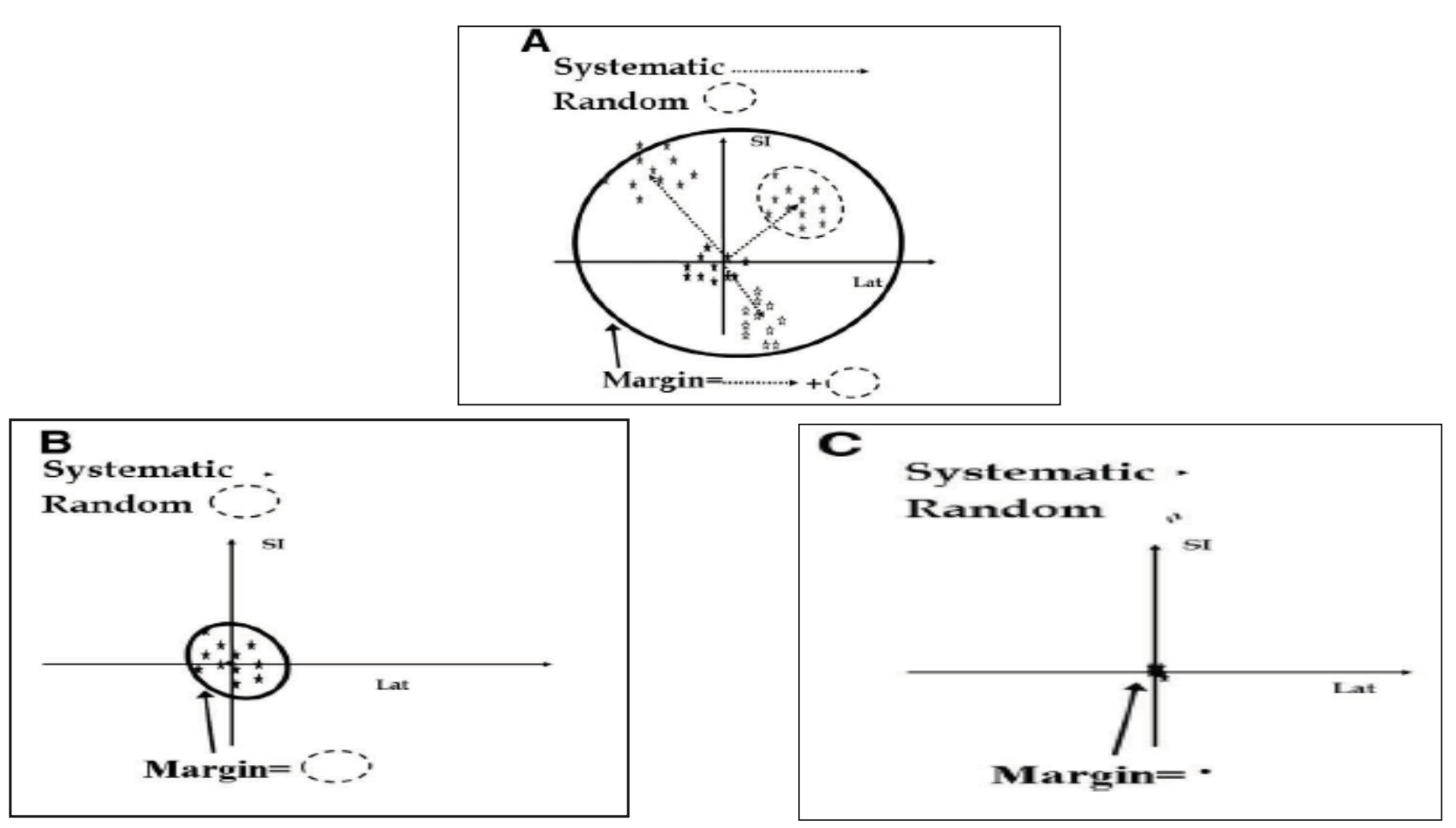

Fig. 1: Definition of how margins (bold large circle) are calculated from systematic (dashed line arrows) and random (dashed line ellipse) errors. Each represent a single localization error. Each group of stars represents observations for a single patient.

(A) Margins from systematic and random errors with no correction.

(B) Margins with systematic errors eliminated because of correction strategy.

(C) Margins with systematic and random errors reduced because of correction.

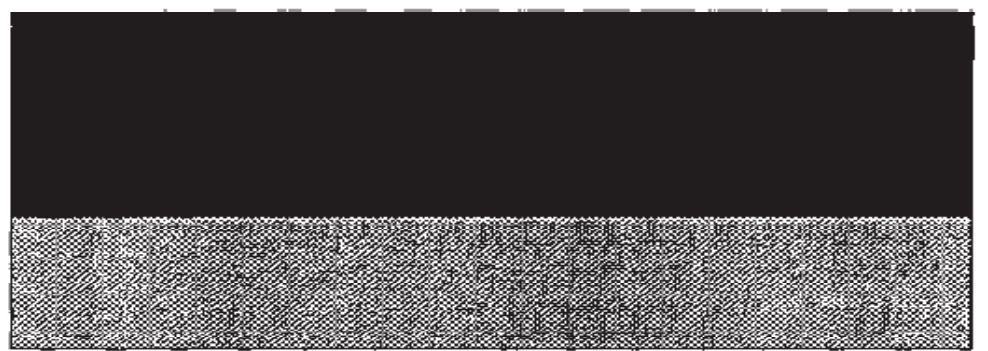

Metal plate (e.g. $1 \mathrm{~mm}$ copper)

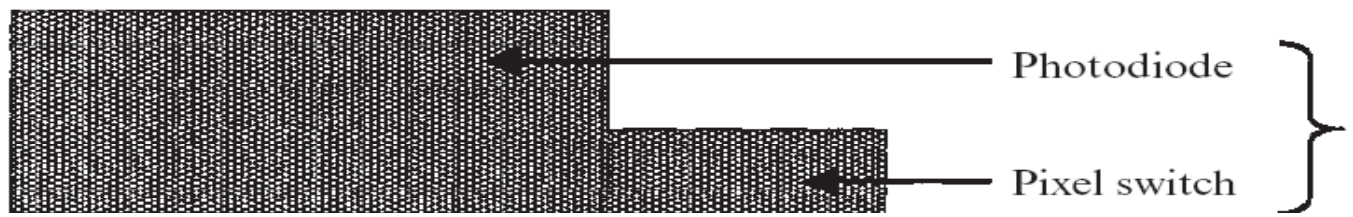

Amorphous silicon

Fig. 2: Cross Sectional Representation of an amorphous silicon electronic portal imaging device. It shows only one pixel. 
In the current work, 10 patients with brain tumors were treated using 3-dimensional conformal radiation therapy technique. Each patient received 25 sessions through 5 weeks. Before the first session, two portal images (Anterior and lateral) were taken and compared with Digital Reconstructed Radiograph (DRR) images transferred from the treatment planning system. These two images were taken to determine the isocenter variations in three dimensions. The variation in each dimension was recorded. The same step was repeated three times per week; i.e. every patient had subjected to 15 electronic portal images with 15 recorded variations for the three dimensions (lateral, vertical and longitudinal dimensions).

\section{Image Comparing Steps:}

1. Digital Reconstructed Radiograph (DRR) was created in the 3 dimensional treatment planning system and transferred to the iView GT.

2. Electronic Portal Image was taken for each patient as a single exposure (2 Monitor Units.

3. Electronic Portal Image was matched with DRR image and stored as a reference image.

4. Each Electronic Portal Image was compared with the first image (Reference Image).

5. Using image analysis tool to assess the variation in 3 dimensions where the differences were recorded

The steps are illustrated in figures 3 a,b,c,d and e ).

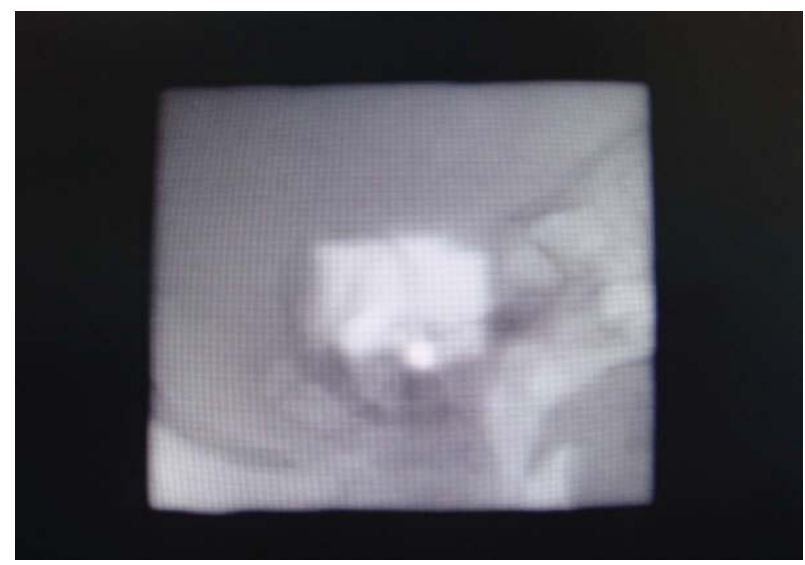

$3 a$

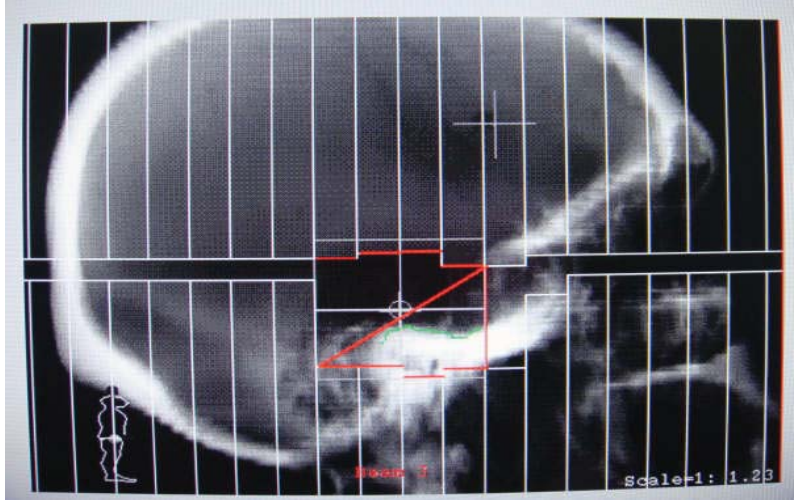

$3 \mathrm{~b}$

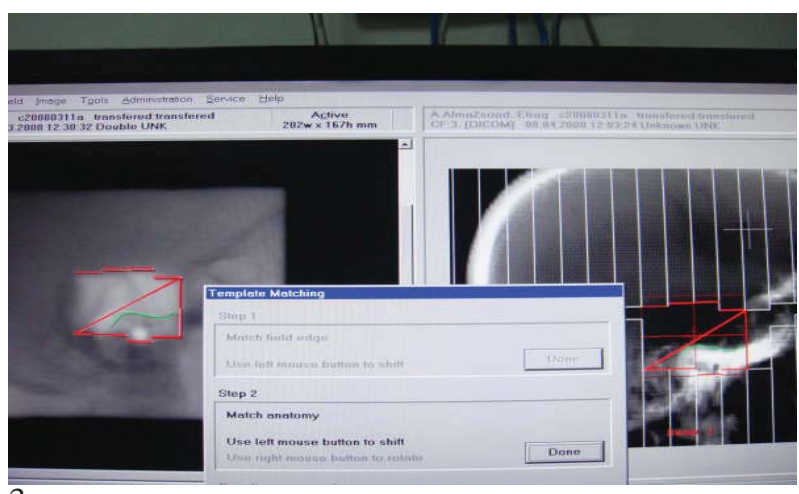

3c

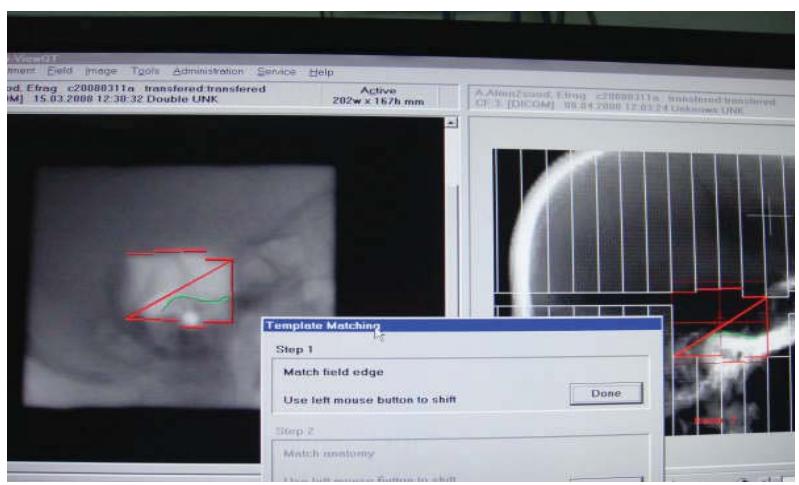

3d

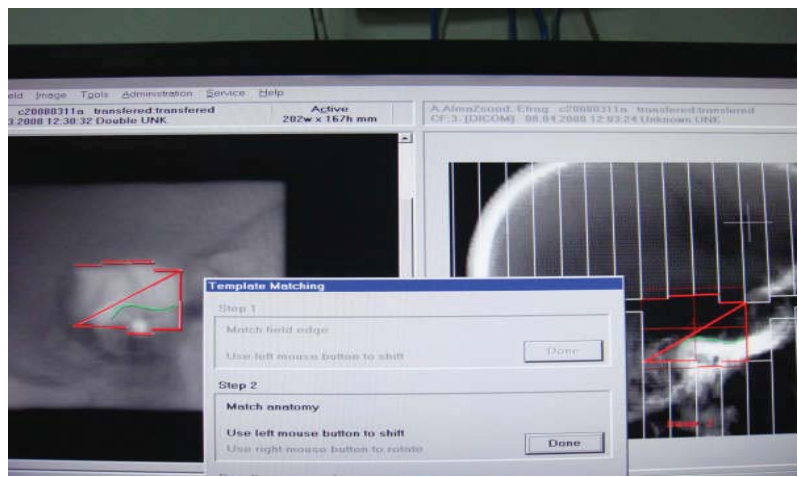

Fig. 3: Illustrating the different Steps of Volume Verification Using EPID.

\section{RESULTS}

Among the studied patients, the average deviation observed is illustrated in Table (1):

Table 1: Avarage Deviations between the Treatment and Setup Isocenters in Millimeters.

\begin{tabular}{lllll}
\hline \multirow{2}{*}{$\begin{array}{l}\text { Patient } \\
\text { Number }\end{array}$} & \multicolumn{2}{l}{ Anterior Field } & \multicolumn{2}{l}{ Lateral Field } \\
\cline { 2 - 5 } & Longitudinal & Lateral & Longitudinal & Vertical \\
\hline 1 & 0.80 & 0.40 & 0.80 & 2.85 \\
2 & 0.73 & 0.80 & 0.73 & 0.40 \\
3 & 1.02 & 0.40 & 1.02 & 0.40 \\
4 & 1.00 & 1.75 & 1.00 & 1.63 \\
5 & 0.78 & 1.65 & 0.78 & 1.55 \\
6 & 2.00 & 3.00 & 2.00 & 1.50 \\
7 & 1.50 & 1.83 & 1.50 & 2.30 \\
8 & 2.50 & 3.00 & 2.50 & 2.00 \\
9 & 2.30 & 2.00 & 2.30 & 4.00 \\
10 & 1.40 & 1.60 & 1.40 & 1.60 \\
\hline \hline
\end{tabular}


The variation was quantified by calculating the systematic and random errors. The variations were ranged from 0.73 to $2.50 \mathrm{~mm}$ longitudinally; 0.40 and up to $3.00 \mathrm{~mm}$ laterally for the anterior fields. For the lateral fields; the variations ranged between 0.73 to $2.50 \mathrm{~mm}$ longitudinally and 0.40 to $4.00 \mathrm{~mm}$ vertically.

\section{DISCUSSION}

Treatment verification is a prerequisite for the verification of complex treatments checking both the treatment planning process and the actual beam delivery. Pretreatment verification can detect errors introduced by the treatment planning systems or differences between planned and delivered dose distributions ${ }^{10}$. The current work was attempted to define margins for the planning target volume definition, accounting for systematic and random setup uncertainties in specified cohort of patients; those with brain tumors planned to receive conformal radiation therapy treatment.

Among the assessed 10 patients, amorphous silicon electronic portal imaging verified systematic and random errors in terms of isocenter shift ranged from 0.73 to 2.50 $\mathrm{mm}$ and 0.40 to 3.00 in both longitudinal and lateral axes ,respectively for the anterior fields, whereas the variations for the isocenter shift longitudinally and vertically ranged from $0.73-2.50$ and $0.40-4.00 \mathrm{~mm}$, respectively for the lateral fields reflecting the extreme accuracy of treatment delivery in relation to the intended planned treatment volumes, and going in agreement with the similar results of van Lin and co-workers, published in $2003^{7}$, where they documented improvement in overall patient setup with reduced variations proposed recipes for clinical and planning clinical and target volumes to 34- mm through evaluating 63 patients with tumor volumes allocated in the head and neck regions over 498 treatment fractions.

On comparing our data to an earlier work by Prisciandaro and colleagues in $2004^{11}$ which indicated lowered values for detected isocenter shifts, where the individual based random error ranged from $(1.1-6.3 \mathrm{~mm})$ and $(1.1-12.3 \mathrm{~mm})$ for anterior and lateral treated fields match on the skull respectively. The superior results documented in our work can be attributed to the smaller number included in our study as well as the impact of including cervical and supraclavicular anatomic locations in the counter study, where its impact upon the variation in isocenter shift yet to be determined.

Portal imaging is a powerful tool in the evaluation of the department specific patient positioning procedures. It can provide direct evidence of treatment variations and thus can demonstrate with confidence the superiority of one technique over the other. The introduction of comfor Table customized head and neck support, in combination with an electronic portal imaging device-based correction protocol have led to an overall improvement in patient setup, while its impact upon long term treatment outcome needs to be better defined in prospective trials to assign its exact role in different treatment allocations.

\section{REFRENCES}

1. Antonuk LE. Electronic portal imaging devices: A review and historical perspective of contemporary technologies and research. Phys.Med.Biol. 2002 Mar 21;47(6):R31-65.

2. Herman MG, Balter JM, Jaffray DA, McGee KP, Munro P, Shalev S, et al. Clinical use of electronic portal imaging: Report of AAPM Radiation Therapy Committee Task Group 58. Med. Phys. 2001 May;28(5):712-37.

3. Clippe S, Sarrut D, Malet C, Miguet S, Ginestet C, Carrie C. Patient setup error measurement using 3D intensity-based image registration techniques. Int.J.Radiat.Oncol.Biol.Phys. 2003 May 1;56(1):259-65.

4. Van Herk M. Errors and margins in radiotherapy. Semin.Radiat. Oncol. 2004 Jan;14(1):52-64.

5. Van Herk M, Remeijer P, Lebesque JV. Inclusion of geometric uncertainties in treatment plan evaluation. Int.J.Radiat.Oncol. Biol.Phys. 2002 Apr 1;52(5):1407-22.

6. Bel A, Keus R, Vijlbrief RE, Lebesque JV. Setup deviations in wedged pair irradiation of parotid gland and tonsillar tumors, measured with an electronic portal imaging device. Radiother. Oncol. 1995 Nov;37(2):153-9.

7. Van Lin EN, van der Vight L, Huizenga H, Kaanders JH, Visser AG. Set-up improvement in head and neck radiotherapy using a 3D off-line EPID-based correction protocol and a customised head and neck support. Radiother.Oncol. 2003 Aug;68(2):137-48.

8. Matsopoulos GK, Asvestas PA, Delibasis KK, Kouloulias V, Uzunoglu N, Karaiskos P, et al. Registration of electronic portal images for patient set-up verification. Phys.Med.Biol. 2004 Jun 21;49(14):3279-89.

9. Herman MG, Pisansky TM, Kruse JJ, Prisciandaro JI, Davis BJ, King BF. Technical aspects of daily online positioning of the prostate for three-dimensional conformal radiotherapy using an electronic portal imaging device. Int.J.Radiat.Oncol.Biol.Phys. 2003 Nov 15;57(4):1131-40.

10. Van Elmpt WJ, Nijsten SM, Dekker AL, Mijnheer BJ, Lambin P. Treatment verification in the presence of inhomogeneities using EPID-based three-dimensional dose reconstruction. Med.Phys. 2007 Jul;34(7):2816-26.

11. Prisciandaro JI, Frechette CM, Herman MG, Brown PD, Garces YI, Foote RL. A methodology to determine margins by EPID measurements of patient setup variation and motion as applied to immobilization devices. Med.Phys. 2004 Nov;31(11):2978-88. 
